\title{
Advance Techniques in Medical Imaging under Big Data Analysis: Covid-19 Images
}

\author{
S. Zimeras \\ Department of Statistics and Actuarial-Financial Mathematics, University of the Aegean, Karlovassi, Samos \\ Email: zimste@aegean.gr
}

How to cite this paper: Zimeras, S. (2021) Advance Techniques in Medical Imaging under Big Data Analysis: Covid-19 Images. Advances in Computed Tomography, 10, 1-10.

https://doi.org/10.4236/act.2021.101001

Received: December 18, 2020

Accepted: March 9, 2021

Published: March 12, 2021

Copyright $\odot 2021$ by author(s) and Scientific Research Publishing Inc. This work is licensed under the Creative Commons Attribution International License (CC BY 4.0).

http://creativecommons.org/licenses/by/4.0/

\section{(c) (i) Open Access}

\begin{abstract}
Quantitative analysis of digital images requires detection and segmentation of the borders of the object of interest. Accurate segmentation is required for volume determination, 3D rendering, radiation therapy, and surgery planning. In medical images, segmentation has traditionally been done by human experts. Substantial computational and storage requirements become especially acute when object orientation and scale have to be considered. Therefore, automated or semi-automated segmentation techniques are essential if these software applications are ever to gain widespread clinical use. Many methods have been proposed to detect and segment 2D shapes, most of which involve template matching. Advanced segmentation techniques called Snakes or active contours have been used, considering deformable models or templates. The main purpose of this work is to apply segmentation techniques for the definition of 3D organs (anatomical structures) when big data information has been stored and must be organized by the doctors for medical diagnosis. The processes would be implemented in the CT images from patients with COVID-19.
\end{abstract}

\section{Keywords}

Segmentation Techniques, Big Data Analysis, Contour Model, Shape Model, Radial Basis Function, Active Contours, Snakes

\section{Introduction}

Segmentation is the process that separates an image into its different parts based on the image characteristics (properties) or the region of interest (organ of interest) that doctors have been considered. Due to the large amount of data that medical images include it is appropriate, a big data analysis to be considered. For 
that reason advance segmentation techniques could be useful to overcome the problem with the data handling.

Segmentation techniques (manual, semi-automatic or automatic) are used in medicine to reproduce the 3D shape structure of the organ (or tumor) that doctor is needed to consider. Many times due to the complexity of the image or shape structure it is not easy to justify the boundaries of the organ manually [1].

In cases where the organs have been moved (due to the body position) or the tumor have been spread inside the body effecting close organs the shape of the region of interest must be segmented very careful due to the importance of the doctor examination. In this case more reflex segmentation techniques must be introduced where they take advantage of the image properties and the elasticity of the potions. These techniques are based on the deformable template defined as active contours or snakes.

The active contour models or Snakes are introduced by a 2D contour model [2] [3] or 3D shape model, in which an initial approximation to the image points or volume voxels are considered and elasticity modeling is taking place by movement of simulated forces (external force). An internal model of the curve is moved considering the neighborhood structure of the images regions (homogeneity). After a starting position is given, the active contour transforms itself to an appropriate contour shape by adapting the external force and internal tension [4] [5] [6]. Snake models are a class of energy minimizing spline curves or surfaces. These models are very important in a number of inverse visual problems such as the segmentation and reconstruction of objects from images in mathematical ill-posed problems.

\section{Main Focus of the Chapter}

\section{Segmentation Techniques}

Segmentation is the process where an image is divided in parts with main goal to justify the particular structure of the region of interest. The technique is used in case a particular organ or a wide area of organs has to be indentified so the structure of them in $2 \mathrm{D}$ or $3 \mathrm{D}$ shape analysis must be represented. In this case the doctors are interested for the shape of the organs, the position inside the body and the anomalies of them. In $2 \mathrm{D}$ structure the presentation of the organ could be a contour model and in 3D structure could be a voxel structure [1].

Mathematically a segmentation is defined as follows [7] [8] [9]: Let $I$ be the set of all image pixels, then by applying segmentation we obtain different unique non-overlapping regions $\left\{S_{1}, S_{2}, \cdots, S_{n}\right\}$ which, when combined, form $I$ :

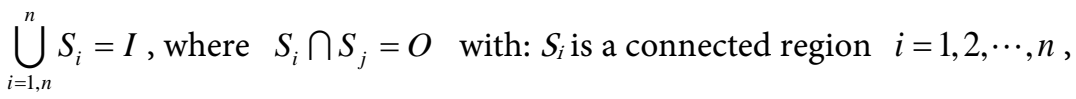

$P\left(S_{i}\right)=$ TRUE for $i=1,2, \cdots, n, P\left(S_{i} \cup S_{j}\right)=$ FALSE for $i \neq j, P\left(S_{i}\right)$ is a logical predicate defined over points in set $S_{i}$. $B_{i}$ are the boundaries in sub-regions $\left\{S_{1}, S_{2}, \cdots, S_{n}\right\}$. So the criteria for image segmentation are given in the following state: 


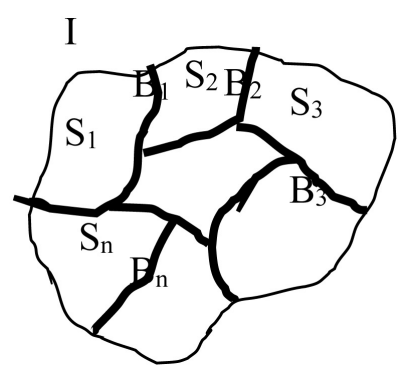

- $B=\bigcup_{i=1}^{n} B_{i}$

- $\quad B_{i} \cap B_{j} \neq \varnothing$, if $S_{i}$ and $S_{j}$ are neighbours

- $S=\left(\bigcup_{i=1}^{n} S_{i}\right) \cup B$

- $S_{i} \cap S_{j}=\varnothing, \forall i, j$,if $i \neq j$

A shape is a connection of points. Planar shapes, represented in terms of a connected set $S \subset R^{2}$ can be mapped by a generic transformation $T$ into its image $Q=T(S)$; example $Q=\operatorname{area}(S)$ (Figure 1 ).

Representation of a shape can be defined as $3 \mathrm{D}$ coordinates of the points on its surface, expressed as $\{x(n), y(n), z(n)\}$, where $n=0,1, \cdots, N-1$ with $N$ the number of points in the surface. In terms of images, a shape could be presented as a $2 \mathrm{D}$ region of interest defining a contour model as $\{x(n), y(n)\}$, where $n=0,1, \cdots, N-1$ with $N$ the number of points in the contour (Figure 2)

Based on the $2 \mathrm{D}$ contours preparation, a $3 \mathrm{D}$ shape of the organ of interested is presented where a surface is defined. A surface is mathematically defined as a 2-Manifold $M$ embedded in $\mathrm{R}^{3}$, where every ball $B$ centered in any point $p$ belonging to the surface, and with a radius $r$ tending to zero, intersects $M$ in a region that is isomorphic to a plane disc [11] [12] [13] (Figure 3).

Considering the Figure 4 it is clear that 2D parallel combined cross-sections illustrate the $3 \mathrm{D}$ shape of the organ. During the segmentation analysis abnormal regions has been appeared into different levels where some of them has been overlapping defining contour orientation models. For that reason a $2 \mathrm{D}$ shape matching approach must be introduced. A set of contours $S_{i}$ and $S_{i+1}$ are calculated to justify the correct contour model.

With the contour orientation calculation, a trees $T_{k, i}$ ( $k$-th tree in $i$-th level) and trees $T_{m, i+1}\left(m\right.$-th tree in $(i+1)$-th level) is calculated and a forest $F_{i}$ and $F_{i+1}$ is defined (Figure 5)

Sets of regions (solid and non-solid) of a level are matched against similar ones on the adjacent level, forming mapping groups mg. The set of all the mapping groups, denoted as MG contains all the mapping groups created for two consecutive levels. In our example the mapping groups for the two levels are $\{(A$, B) $\}$ vs $\{(1,2),(3,4)\}$.

After the orientation of the contours an interpolation procedure must be established when parallel cross-sections contours are used. Based on the interpolation procedure a $3 \mathrm{D}$ shape of the organ can be illustrated and surface model can 

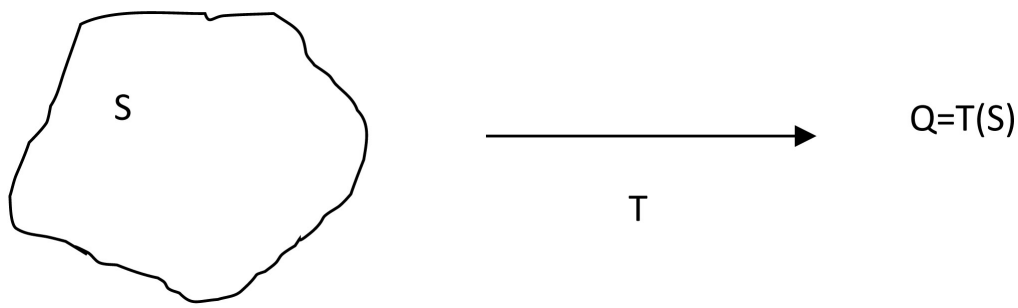

Figure 1. Shape model.

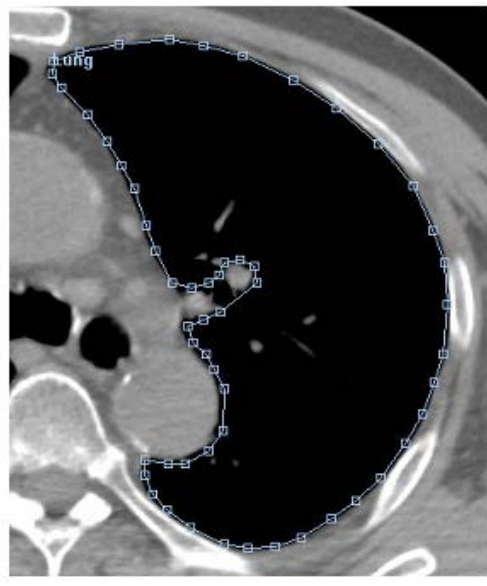

Figure 2. Contour model [8] [10].
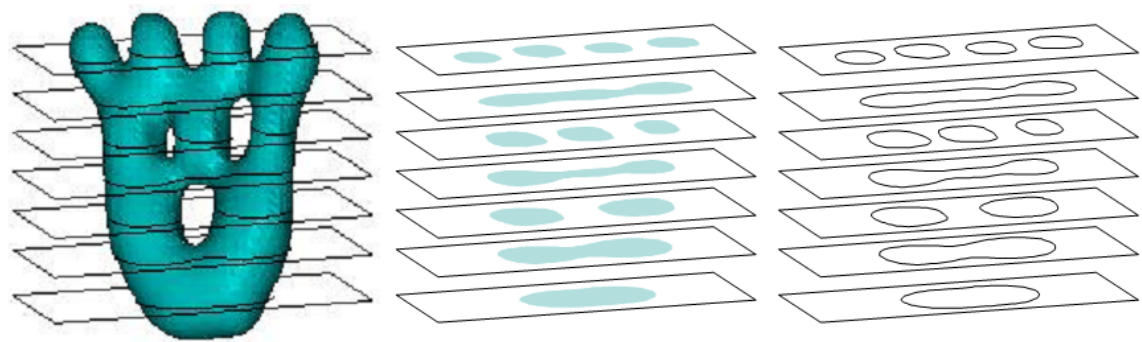

Figure 3. Parallel levels from a simple object [14].

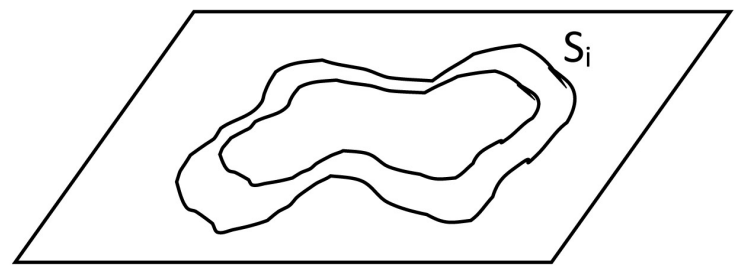

$\mathrm{L}_{\mathrm{i}}$

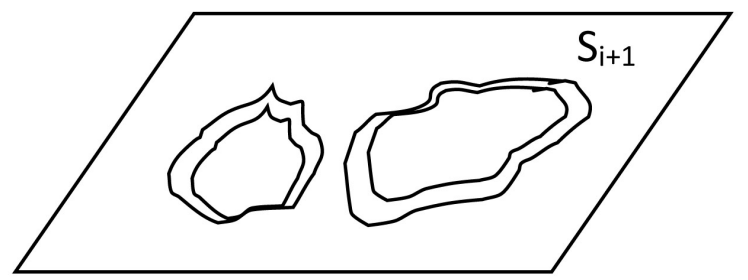

$\mathrm{L}_{\mathrm{i}+1}$

Figure 4. Contour orientations in different levels. 


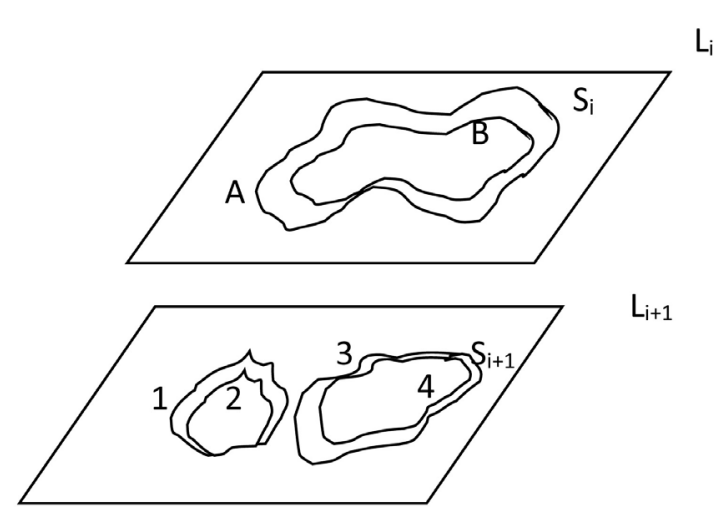

$L_{i}$

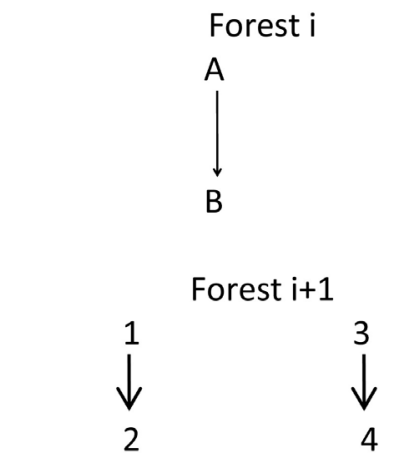

Figure 5. Contour calculation in different levels.

be calculated. Interpolation procedures are used in case when anomalies of the contours between different levels are large. The approach that is proposed is based on [15] [16] [17] considering a radial basis interpolates function to generate implicit surfaces.

\section{Solutions and Recommendations}

For the 3D surface interpolation the contours between different slices are appeared to be abnormal. Meaning the in the plane of slice $i$, the surface created between slice pairs $i-1$ and $i$ will usually not agree in surface normal with the surface created between slices I and $i+1$. To avoid discontinuities in surface normal, we must use information that involves more than just two slices at a given time. The contour interpolation result between parallel contours (using 10 contours producing initial 600 triangles) is illustrated in Figure 6.

The most of the times using segmentation techniques a serious problem is the shape of the organ for the $3 \mathrm{D}$ reconstruction. When there are not a lot of a anomalies (convex shape) traditional segmentation techniques like boundary tracking or region growing could be used effectively to reconstruct the organ of interest. In case that there are a few anomalies (concave shape) in this case new segmentation techniques must be considered [18] [19] [20]. [21] reported a method based on an adaptation of 3D deformable surface models to the boundaries of the anatomic structures of interest.

Active contours or snakes are part of deformable models. The idea behind these models is to represent the contours as parts of elasticity and rigidity. The general concept of active contours is autonomous adaptation of the shape and location of objects finding the important contour points to reconstruct the image. After initialization, by a starting contour, the process performs a fitting process based on the elasticity of the contour lines. If the model is represented by a parametric curve in $2 \mathrm{D} v(s)=(x(s), y(s))$. For each point in the neighborhood of $v_{i}$ an energy function is calculated

$$
E_{i}=a E_{\text {int }}\left(v_{i}\right)+\beta E_{\text {ext }}\left(v_{i}\right)
$$

where $E_{\text {int }}$ is the energy function dependent on the shape of the contour and $E_{\text {ext }}$ 


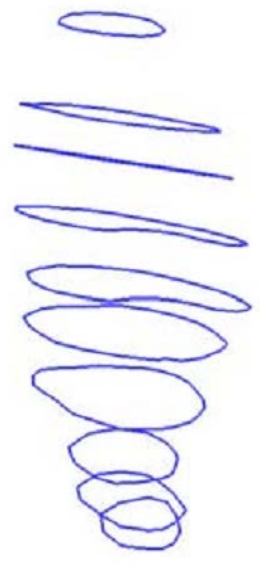

(a)

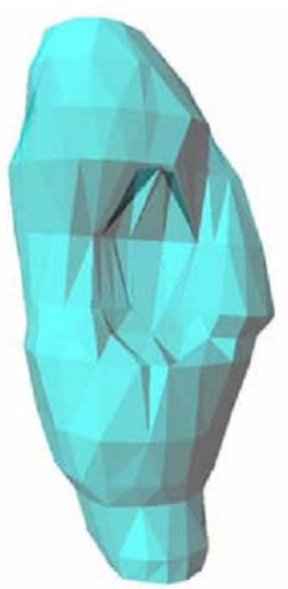

(b)

Figure 6. Reconstruction of kidney surface from parallel contours. In (a) manual segmented contours (b) the implicit surface interpolation algorithm [8] [10].

is the energy function dependent on the image properties. Based on [2] a generalized active contour model is proposed based on

$$
E=\int_{0}^{1} E\left(v_{r}\right)=\int_{0}^{1}\left(E_{\text {int }}\left(v_{r}\right)+E_{\text {image }}\left(v_{r}\right)\right)+E_{\text {ext }}\left(v_{r}\right) \mathrm{d} r
$$

where $E_{\text {int }}$ is the internal energy of the model and characterizes the smoothness of the curve, $E_{\text {image }}$ is the image energy and characterizes of the curve and $E_{\text {ext }}$ is the external energy model and characterizes the user interaction into the model. The corresponding proposed models for the above energy functions are given bellow [2] [4]:

- For internal energy: $E_{\text {int }}\left(v_{r}\right)=a_{1}(r)\left|\frac{\partial v}{\partial r}\right|^{2}+a_{2}(r)\left|\frac{\partial^{2} v}{\partial r^{2}}\right|^{2}$, where the first derivative represents the elasticity of the spline and the second derivative represents the bending of the contour.

- For image energy: $E_{\text {image }}\left(v_{r}\right)=\beta_{1} E_{\text {line }}\left(v_{r}\right)+\beta_{2} E_{\text {edge }}\left(v_{r}\right)+\beta_{3} E_{\text {curve }}\left(v_{r}\right)$, where $E_{\text {line }}\left(v_{r}\right)=I\left(v_{r}\right)$, brightness of the image, $E_{\text {edge }}\left(v_{r}\right)=-\left|\nabla I\left(v_{r}\right)\right|^{2}$ stepping of the image and $E_{\text {curve }}\left(v_{r}\right)=\frac{I_{v v}\left(v_{r}\right)}{I_{w}\left(v_{r}\right)}$, corners of the regions

- For the external energy: $E_{\text {exter }}\left(v_{r}\right)=\gamma_{1}\left(v_{r_{i}}-x_{i}\right)^{2}$ where depending of the sign of $\gamma$, the curve is either attracted or repelled from the user defined point. Figure 7 illustrates the original CT image example with patient diagnoses with COVID-19. The region with uncertain diagnose is given in the square shape. The second image illustrates the resulting sub region and the third image illustrates a binary image based on the region gowning algorithm [21].

The two algorithms that was considered for analysis is based on [2] and a modification based on the [22] [23]. Results for the process are given in Figure 8. The segmented regions with uncertain diagnose are given based on the final contours. Part of the analysis has been implemented based on [24]. 


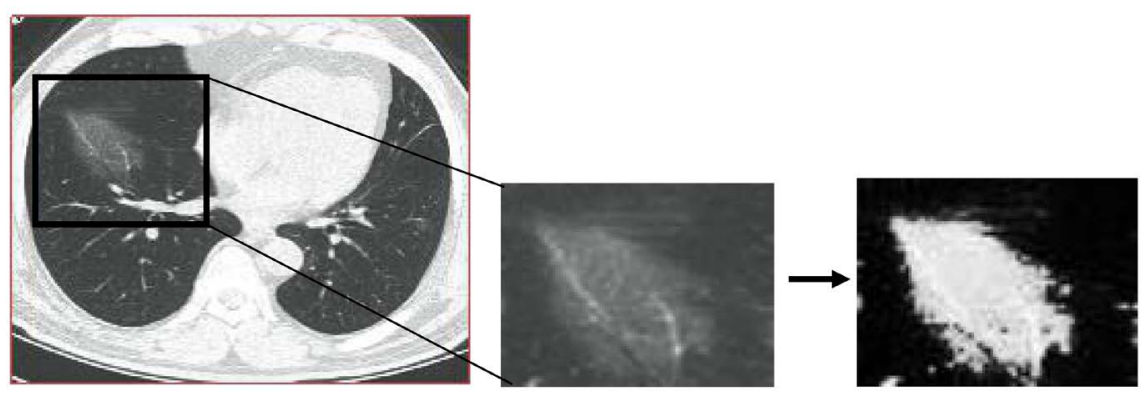

Figure 7. Examples of the original CT patient images with COVID-19. From left to right: Original image, sub region of interest, binary image using region growing algorithm.
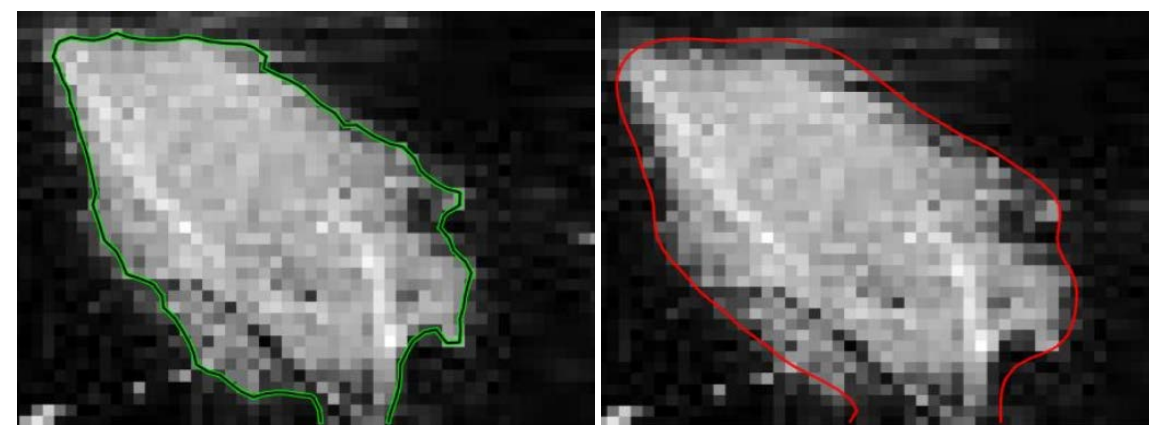

Figure 8. Example of the snake algorithm applied to CT images of COVID-19 segmentation. From left to right: Segmented image based on [2]; Segmented image based on [22] [23].

From Figure 8 is clear that the regions with the medical problems are indentified clearly in the applied method considering active contours analysis. Especially the small regions in the original image are presented quit clearly in the reproductive results.

Considering the algorithms, the converges for the final solution is achieved with 100 iteration for [2] and 117 iterations for [22]; calculation time $0.654 \mathrm{sec}$ for [2] and $0.670 \mathrm{sec}$ for [22]. As similarity measure is considered the Dice criterion with form

$$
\text { Dice }=\frac{2(A \cap B)}{A+B}
$$

where $A$ and $B$ are the segmented region from method 1 and the segmented region from method 2. If $A \approx B$ then Dice $\rightarrow 1$. If $A=B$ then Dice $=1$ (same segmentation contour results). In our comparison the Dice measure is 0.907 meaning that both methods give us approximate similar results.

\section{Future Research Directions}

Further direction of this work could be the improvement of segmentation techniques especially for the $3 \mathrm{D}$ shape models. In this case a new volumetric model must be considered based on the anomalies of the surface reconstruct of the organ. An approach could be an introduction of stochastic models based on the homogeneity of the neighborhood structure. 
Until now for 2D models a Markov chain techniques has been introduced considering the local characteristics of the image leading us to Markov Random Fields modeling (homogeneous or inhomogeneous). For the 3D models an appropriate model could be introduced based on the local characteristics of the voxels and a 3d-Markov Random Fields model could be used reforming the neighborhood structure model (from 2D to 3D) [25] [26] [27]

Estimation of the model parameters could be done by proposing deterministic (maximum likelihood procedures) or stochastic (Monte Carlo Markov Chains procedures) to overcome possible problems with the stability of the estimation [25] [26] [27] [28]

\section{Conclusions}

Quantitative analysis of medical images requires detection and segmentation of the borders of the object of interest. Medical images segmentation techniques are part of big data analysis and for that reason different approaches must be considered due to the structure of the organ that the doctors needs to examine.

In medical images, segmentation has traditionally been done by human experts. Substantial computational and storage requirements become especially acute when object orientation and scale have to be considered. Considering the shape model of the organ many times it is difficult for the doctors to segment by hand the appropriate regions. For that reason advances segmentation techniques could be proposed to overcome this particular problem.

Many methods have been proposed to detect and segment 2D shapes, the most of which is template matching. Advanced segmentation techniques called snakes or active contours have been used, considering deformable models or templates. These techniques are based on the properties of the image (brightness, sharpness, and boundaries) for the final reconstruction of the shape contour. To problem with the anomalies between different cross-section contours models under parallel positions could be solved by proposing a radial basis function model where an interpolation method is proposed to manage the overlapping between the mapping groups.

\section{Conflicts of Interest}

The author declares no conflicts of interest regarding the publication of this paper.

\section{References}

[1] Kuszyk, B.S., Ney, D.R. and Fishman, E.K. (1995) The Current State of the Art in Three Dimensional Oncologic Imaging: An Overview. International Journal of Radiation Oncology, Biology, Physics, 33, 1029-1039. https://doi.org/10.1016/0360-3016(95)02003-9

[2] Kass, M., Witkin, A. and Terzopoulos, D. (1987) Snakes: Active Contour Models. IEEE First International Conference on Computer Vision, London, 8-11 June 1987, 259-268. 
[3] Blake and Isard, M. (1998) Active Contours: The Application of Techniques from Graphics, Vision, Control Theory and Statistics to Visual Tracking of Shapes in Motion. Springer-Verlag, London.

[4] Cinerney, M.T. and Terzopoulos, D. (1995) Topologically Adaptable Snakes. 5th International Conference on Computer Vision, Cambridge, 20-23 June 1995, 840845.

[5] Sakas, G., Karangelis, G. and Pommert, A. (2001) Advanced Applications of Volume Visualization Methods in Medicine. In: Stergiopoulos, S., Ed., Advanced Signal Processing Handbook, Theory and Implementation for Radar, Sonar, and Medical Imaging Real- Time Systems, CRC Press, Boca Raton.

[6] Behr, J., Choi, S.M., Großkopf, S., Hong, H., Nam, S.A., Peng, Y., Hildebrand, A., Kim, M.H. and Sakas, G. (2000) Modeling, Visualization, and Interaction Techniques for Diagnosis and Treatment Planning in Cardiology. Computer \& Graphics, 24, 741-753. https://doi.org/10.1016/S0097-8493(00)00076-5

[7] Raut, S., Raghuvanshi, M., Dharaskar, R. and Raut, A. (2009) Image Segmentation-A State-of-Art Survey for Prediction. In: Proceedings of International Conference on Advanced Computer Control, IEEE Computer Society, Singapore, 420424.

[8] Zimeras, S., Gortzis, L. and Pylarinou, Ch. (2013) Shape Analysis in Radiotherapy and Tumor Surgical Planning Using Segmentation Techniques. In: System-Level Design Methodologies for Telecommunication, Springer, Berlin, 159-173. https://doi.org/10.1007/978-3-319-00663-5 9

[9] Gonzales, R. and Woods, R. (2001) Digital Image Processing. 2nd Edition, Prentice Hall, Upper Saddle River.

[10] Karangelis, G. (2004) 3D Simulation of External Beam Radiotherapy. Ph.D. Thesis, University of Darmstadt, Darmstadt.

[11] Ruiz, O. (2002) Understanding CAD/CAM/CG. American Society of Mechanical Engineers ASME, Continuing Education Institute, Global Training, ASME Code GT-006.

[12] Ruiz, O. and Cadavid, C. (2001) Boolean 2d Shape Similarity for Surface Reconstruction. Visualization, Imaging and Image Processing (VIIP 2001), Marbella, 3-5 September 2001, 1-11.

[13] Ruiz, O., Cadavid, C. and Granados, M. (2001) Evaluation of 2D Shape Likeness for Surface Reconstruction. XIII International Congress on Graphics Engineering, Badajoz, June 2001, 16-24.

[14] Osorio, E.M.V. (2003) Surface Reconstruction Diploma Thesis, Universidad Eafit Escuela de Ingenieria Departemento de Informatica Y Sistemas Medellin.

[15] Turk, G. and O’Brien, J.F. (2002) Modelling with Implicit Surfaces That Interpolate. ACM Transaction on Graphics, 21, 855-873. https://doi.org/10.1145/571647.571650

[16] Turk, G. and O'Brien, J.F. (1999) Shape Transformation Using Variation Implicit Functions. Proceedings of the 26th Annual Conference on Computer Graphics and Interactive Techniques, Los Angeles, July 1999, 335-342.

https://doi.org/10.1145/311535.311580

[17] Turk, G. and O’Brien, J.F. (1999) Variation Implicit Surfaces. Tech Report GITGVU-99-15, Georgia Institute of Technology.

[18] Karangelis, G., Zimeras, S., Firle, E., Wang, M. and Sakas, G. (2001) Volume Definition Tools for Medical Image Applications. In: Viergever, M.-A., Dohi, T. and Vannier, M., Eds., 4th MICCAI International Conference, Springer-Verlag, Utrecht, 
Lecture Notes in Computer Sciences 2208, 1295-1297.

https://doi.org/10.1007/3-540-45468-3 201

[19] Karangelis, G. and Zimeras, S. (2002) An Accurate 3D Segmentation Method of the Spinal Canal Applied on CT Images. In: BVM 2002 Conference Proceedings, Springer-Verlag, Berlin, 366-369. https://doi.org/10.1007/978-3-642-55983-9 87

[20] Karangelis, G. and Zimeras, S. (2002) 3D Segmentation Method of the Spinal Cord Applied on CT Data. Computer Graphics Topics, 14, 28-29. https://doi.org/10.1007/978-3-642-55983-9 87

[21] Zimeras, S. (2019) Brain Segmentation Tools under Uncertain Conditions for Radiotherapy Treatment Planning, Biomedical Research and Clinical Practice, 4, 1-5. https://doi.org/10.15761/BRCP.1000183

[22] Chan, T.F. and Vese, L.A. (1999) An Active Contour Model without Edges. In: Nielsen, M., Johansen, P., Fogh Olsen, O. and Weickert, J., Eds., Scale-Space 1999, Springer, Berlin, Lecture Notes in Computer Science, Vol. 1682, 141-151. https://doi.org/10.1007/3-540-48236-9 13

[23] Chan, T.F. and Vese, L.A. (2001) Active Contours without Edges. IEEE Transactions on Image Processing, 10, 266-277. https://doi.org/10.1109/83.902291

[24] Nikolay, S. (2021) Active Contours Implementation \& Test Platform GUI.

[25] Aykroyd, R.G. and Zimeras, S. (1999) Inhomogeneous Prior Models for Image Reconstruction. Journal of American Statistical Association, 94, 934-946. https://doi.org/10.1080/01621459.1999.10474198

[26] Zimeras, S. and Matsinos, Y. (2019) Modeling Uncertainty Based on Spatial Models in Spreading Diseases: Spatial Uncertainty in Spreading Diseases. International Journal of Reliable and Quality E-Healthcare, 8, 55-66. https://doi.org/10.4018/IJRQEH.2019100103

[27] Zimeras, S. (1997) Statistical Models in Medical Image Analysis. Ph.D. Thesis, University of Leeds, Leeds.

[28] Zimeras, S. (2006) Simulating Texture Patterns Using Auto-Logistic Models. WSEAS Transactions on Systems, 5, 2269-2276. 